\title{
Rectal tuberculosis after infliximab therapy despite negative screening for latent tuberculosis in a patient with ulcerative colitis
}

\author{
Jatinderpal Singh ${ }^{1}$, Amarender S Puri ${ }^{1}$, Sanjeev Sachdeva ${ }^{1}$, Puja Sakhuja ${ }^{2}$, Kulandaivelu Arivarasan ${ }^{1}$ \\ Departments of ${ }^{I}$ Gastroenterology and ${ }^{2}$ Pathology, GB Pant Institute of Postgraduate Medical Education and Research (GIPMER), New Delhi, India
}

Tumor necrosis factor- $\alpha$ inhibitors are now considered as standard therapy for patients with severe inflammatory bowel disease who do not respond to corticosteroids, but they carry a definite risk of reactivation of tuberculosis. We present a case in which a patient with inflammatory bowel disease developed a de novo tuberculosis infection after the start of anti-tumor necrosis factor- $\alpha$ treatment despite showing negative results in tuberculosis screening. Although there are many case reports of pleural, lymph nodal and disseminated tuberculosis following infliximab therapy, we present the first case report of rectal tuberculosis following infliximab therapy. (Intest Res 2016;14:183-186)

Key Words: Inflammatory bowel diseases; Colitis, ulcerative; Tumor necrosis factor-alpha; Tuberculosis; Adverse event

\section{INTRODUCTION}

Tumor necrosis factor- $\alpha$ (TNF- $\alpha$ ) inhibitors are now considered part of the standard care for patients with severe ulcerative colitis (UC) who do not respond to corticosteroids. ${ }^{1}$ TNF- $\alpha$ is also critical for the formation of tuberculous granulomas. ${ }^{2}$ Therefore TNF- $\alpha$ inhibitors are associated with a definite risk of reactivation of tuberculosis (TB). ${ }^{3} \mathrm{In}$ view of the risk of reactivation of latent $\mathrm{TB}$, screening for $\mathrm{TB}$ before starting anti TNF- $\alpha$ therapy has become mandatory. ${ }^{4}$ At present, screening for latent TB consists of a thorough history along with a tuberculin skin test (TST), interferon- $\gamma$ release assay (IGRA), and chest radiography. ${ }^{4}$ Herein we report one patient who developed disseminated TB with involvement of the rectum during the course of anti TNF- $\alpha$ therapy, despite negative screening for latent TB.

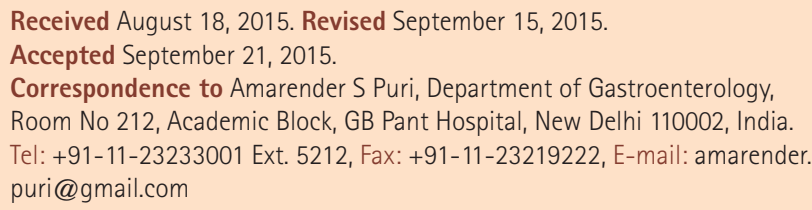

Financial support: None. Conflict of interest: None.

\section{CASE REPORT}

A 38-year old man was diagnosed with UC in 2012. At presentation, his composite Mayo score was 8/12 (Fig. 1); the disease extent revealed by colonoscopy was E3 according to the Montreal classification. ${ }^{5}$ The patient was initially treated with mesalamine followed by a combination of oral steroids along with azathioprine $(100 \mathrm{mg} / \mathrm{d})$ for active disease. Owing to persistent disease activity in January 2014, he was offered the choice of anti TNF- $\alpha$ therapy. There was no past history of $\mathrm{TB}$ or any history of recent contact with anyone with active TB. The TST yielded negative results with an induration of $3 \mathrm{~mm}$; IGRA was also negative with a value of $0.09 \mathrm{IU} / \mathrm{mL}$ for the antigen tube and $0.03 \mathrm{IU} / \mathrm{mL}$ for the nil tube. His chest radiograph was also normal. Infliximab (5 mg/kg) was started in January 2014, and treatment with azathioprine and mesalamine continued. After the first two doses, the patient went into complete remission with a Mayo score of 2/12. In October 2014, after six doses of infliximab, the patient started complaining of pain in the left side of his chest along with an increase in the frequency of stools to 5 to 6 per day and 1 to 2 per night, although there was no blood

\footnotetext{
(c) Copyright 2016. Korean Association for the Study of Intestinal Diseases. All rights reserved.

This is an Open Access article distributed under the terms of the Creative Commons Attribution Non-Commercial License (http://creativecommons.org/licenses/by-nc/4.0)

which permits unrestricted non-commercial use, distribution, and reproduction in any medium, provided the original work is properly cited.
} 


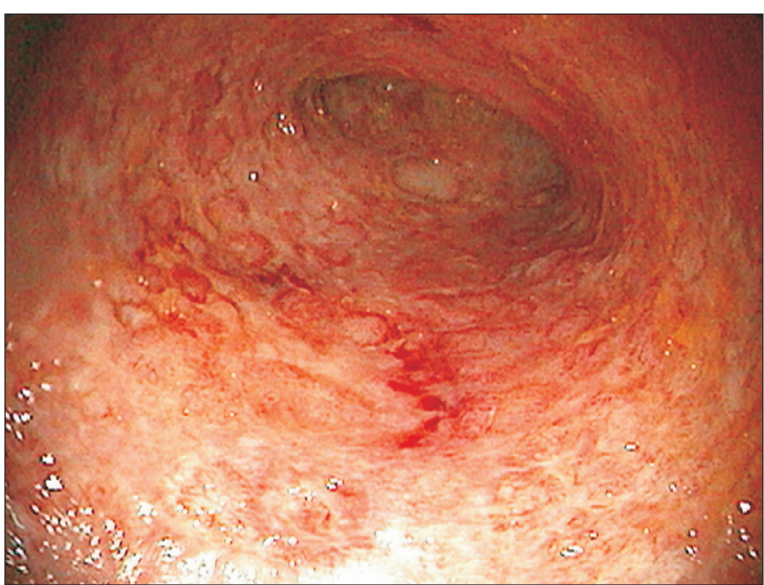

Fig. 1. Colonoscopic finding. Colonoscopic image of the patient showing moderate disease according to the Mayo score (marked erythema, lack of vascular pattern, friability, erosions and superficial ulcers).

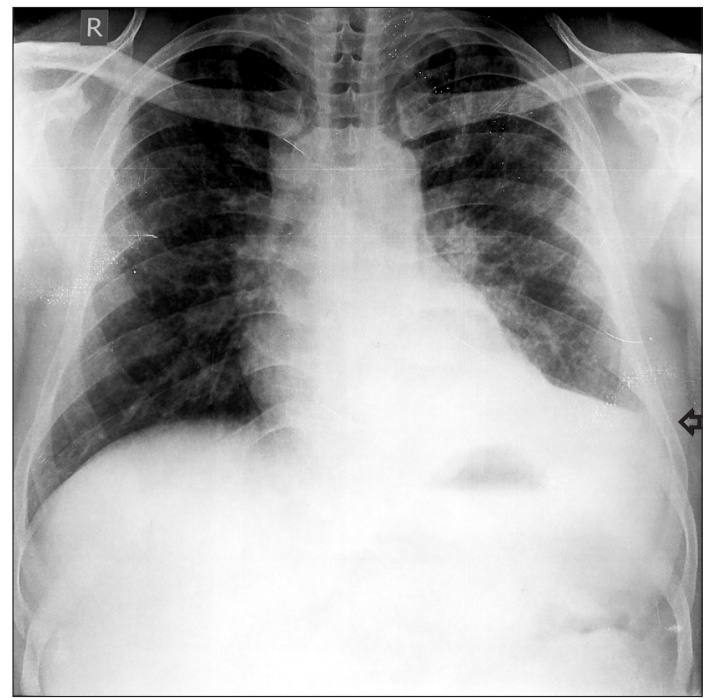

Fig. 2. Chest X-ray finding. Chest radiograph showing left-sided pleural effusion (arrow).

in the stools.

Chest radiography showed left-sided pleural effusion (Fig. 2). Biochemical analysis of the pleural fluid showed a serumpleural fluid albumin gradient of 0.7 and an adenosine deaminase value of $93 \mathrm{IU} / \mathrm{mL}$. Cytological examination of the fluid showed 143 cells (90\% lymphocytes). Sigmoidoscopy revealed a large, single punched out ulcer $(20 \times 10 \mathrm{~mm})$ in the rectum (Fig. 3) with the surrounding mucosa showing a normal vascular pattern. A biopsy specimen from the rectal ulcer showed epithelioid cell granulomas (Fig. 4) without any caseating necrosis. No acid-fast bacilli were demonstrated on Ziehl-Nielsen staining of the rectal biopsy specimen. A diagnosis of infliximab induced disseminated TB was

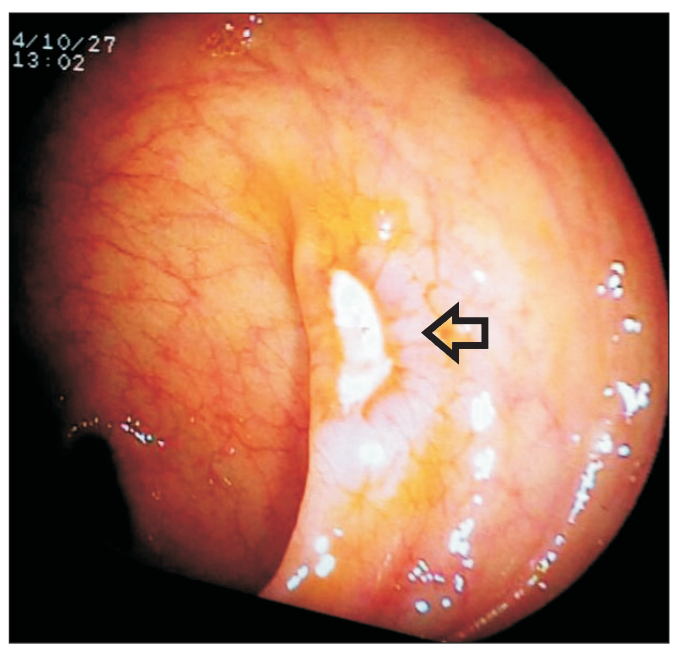

Fig. 3. Sigmoidoscopic finding. Tubercular rectal ulcer (arrow) in the background of almost normal colonic mucosa.

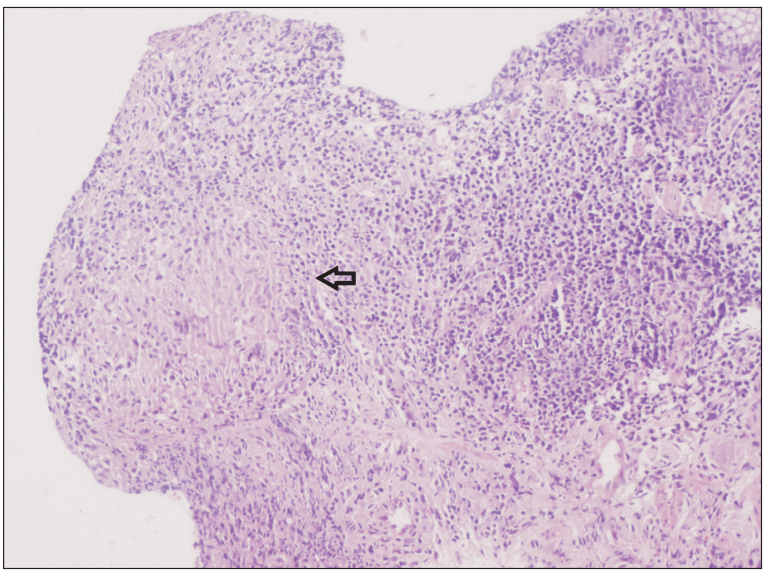

Fig. 4. Histopathological finding. Histopathology from the rectal ulcer shows well-defined granuloma with epithelioid cells (arrow) $(\mathrm{HEE}, \times 10)$.

made, and anti TNF- $\alpha$ therapy was stopped. The patient was started on a four-drug regimen consisting of streptomycin, isoniazid, rifampicin and pyrazinamide in November 2014. After 3 months of anti-tubercular therapy, there was complete resolution of the pleural effusion (Fig. 5), along with complete healing of the rectal ulcer. The patient was continued on azathioprine and mesalamine after the discontinuation of infliximab without any clinical relapse.

\section{DISCUSSION}

Keane et al. ${ }^{3}$ were the first to establish a relationship between the reactivation of TB following the use of anti TNF- $\alpha$ inhibitors for inflammatory arthritis or IBD. Their report highlighted the fact that nearly a quarter of these patients had disseminated TB with a high case fatality ratio of $17 \%$. 


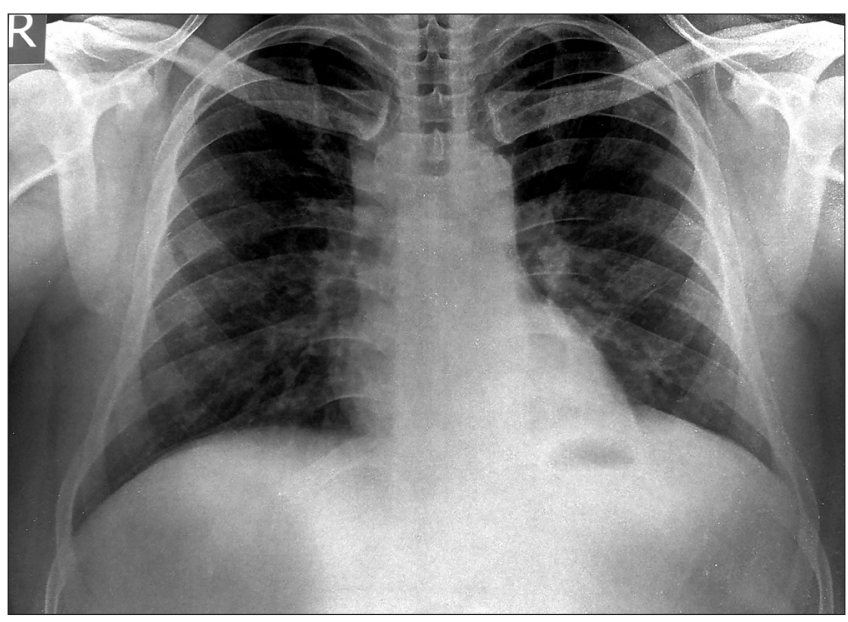

Fig. 5. Chest $X$-ray finding. Complete resolution of pleural effusion on chest radiography after antitubercular therapy.

Extra-pulmonary disease was documented in $57 \%$ of these patients, with lymph nodes being the commonest extrapulmonary site. The median time to detection of TB was 12 weeks from the administration of the first dose of infliximab. ${ }^{3}$ Korean data suggests that the risk of reactivation of TB following biological therapy is higher in patients with IBD than in those with rheumatological diseases. After adjusting for age and sex, the incidence rate ratio for IBD patients to develop TB was 5.97 compared with 1.02 for rheumatoid arthritis patients. ${ }^{6}$ The median time to occurrence of TB in the Korean study was 5.4 months, which is some-what longer than in the original report by Keane et al. ${ }^{3}$ Byun et al. ${ }^{7}$ showed that patients exposed to TNF- $\alpha$ inhibitors develop TB with an adjusted risk ratio of 11.7 with $20 \%$ developing extrapulmonary TB. Another study by the same author reported 25 cases of TB in a cohort of 873 IBD patients, 19 of whom screened negative for latent $\mathrm{TB}$, with a mean duration of 25.6 months after initiation of anti TNF- $\alpha$ therapy. ${ }^{8}$

In our patient, latent TB was excluded by a normal chest radiography, negative TST, and a negative IGRA before commencement of the biologic therapy. Additionally, there was no history of TB. After an initial dramatic response to infliximab, he had a recurrence of diarrhea 5 months after the initiation of infliximab therapy due to isolated rectal TB as the rest of the colon did not show any other lesion.

The rectum is an uncommon site of TB even in countries where the disease is endemic. The commonest symptoms are rectal bleeding and diarrhea. The typical morphological appearance is a concentric stricture as seen on sigmoidoscopy. ${ }^{9}$ A correct diagnosis is possible on biopsies obtained at sigmoidoscopy, although in some patients, the correct diag- nosis is made only after surgical resection of the rectum for suspected rectal malignancy. ${ }^{9}$

This case illustrates that rectal involvement may be seen as a part of disseminated TB in patients with IBD following anti TNF- $\alpha$ therapy. As has been highlighted by other reports, TB may occur following exposure to infliximab even in patients with no evidence of latent $\mathrm{TB} .{ }^{10,11}$ As this case illustrated, rectal lesions are most amenable to biopsy and offer the opportunity for making a definite diagnosis of TB. To the best of our knowledge, rectal TB has not previously been reported as a consequence of infliximab therapy in IBD. In summary, the recurrence of diarrhea after the initial response to infliximab therapy in patients with UC warrants a colonoscopy to exclude the diagnosis of colorectal TB.

\section{REFERENCES}

1. Rutgeerts P, Sandborn WJ, Feagan BG, et al. Infliximab for induction and maintenance therapy for ulcerative colitis. N Engl J Med 2005;353:2462-2476.

2. Fallahi-Sichani M, El-Kebir M, Marino S, Kirschner DE, Linderman JJ. Multiscale computational modeling reveals a critical role for TNF-alpha receptor 1 dynamics in tuberculosis granuloma formation. J Immunol 201 1;186:3472-3483.

3. Keane J, Gershon S, Wise RP, et al. Tuberculosis associated with infliximab, a tumor necrosis factor alpha-neutralizing agent. N Engl J Med 2001;345:1098-1104.

4. Shim TS. Diagnosis and treatment of latent tuberculosis infection in patients with inflammatory bowel diseases due to initiation of anti-tumor necrosis factor therapy. Intest Res 2014;12:12-19.

5. Satsangi J, Silverberg MS, Vermeire S, Colombel JF. The Montreal classification of inflammatory bowel disease: controversies, consensus, and implications. Gut 2006;55:749-753.

6. Jung SM, Ju JH, Park MS, et al. Risk of tuberculosis in patients treated with anti-tumor necrosis factor therapy: a nationwide study in South Korea, a country with an intermediate tuberculosis burden. Int J Rheum Dis 2015;18:323-330.

7. Byun JM, Lee CK, Rhee SY, et al. The risk of tuberculosis in Korean patients with inflammatory bowel disease receiving tumor necrosis factor-alpha blockers. J Korean Med Sci 2015;30:173-179.

8. Byun JM, Lee CK, Rhee SY, et al. Risks for opportunistic tuberculosis infection in a cohort of 873 patients with inflammatory bowel disease receiving a tumor necrosis factor-alpha inhibitor. Scand J Gastroenterol 2015;50:312-320.

9. Puri AS, Vij JC, Chaudhary A, et al. Diagnosis and outcome of isolated rectal tuberculosis. Dis Colon Rectum 1996;39:11261129 
10. Debeuckelaere C, De Munter P, Van Bleyenbergh P, et al. Tuberculosis infection following anti-TNF therapy in inflammatory bowel disease, despite negative screening. J Crohns Colitis 2014;8:550-557.
11. O’Dowd C, Kewin P, Morris J, Cotton M. Tuberculosis complicated by immune reconstitution inflammatory syndrome in a patient on anti-TNF $\alpha$ therapy for Crohn's disease. BMJ Case Rep 2011;2011:bcr0920103376. doi:10.1136/bcr.09.2010.3376. 